\title{
Domínios e temáticas no campo das práticas hospitalares em terapia ocupacional: uma revisão da literatura brasileira de 1990 a 2006*
}

\author{
Domains and themes in the field of occupational \\ therapy in hospital practice: a literature review of \\ Brazilian production from 1990 to 2006
}

\author{
Sandra Maria Galheigo ${ }^{1}$
}

\begin{abstract}
GALHEIGO, S. M. Domínios e temáticas no campo das práticas hospitalares em terapia ocupacional: uma revisão da literatura brasileira de 1990 a 2006. Rev. Ter. Ocup. Univ. São Paulo, v.18, n. 3, p. 113-121, set./dez. 2007.

RESUMO: Este artigo se propõe a apresentar a produção do conhecimento no campo das práticas hospitalares em terapia ocupacional a partir de seus domínios e temáticas, através de estudo da produção científica brasileira de 1990 a 2006. Foi analisada a produção na forma de artigos de periódicos, capítulos de livro e resumos ampliados dos Anais de dois Congressos Brasileiros de Terapia Ocupacional, o que resultou num levantamento bibliográfico de 83 registros. O material analisado foi organizado em quatro domínios de atenção: gestante, puérpera e neonato; criança e adolescente em enfermarias pediátricas; adulto e idoso em hospital geral; pessoas com câncer e/ou HIV/AIDs e; fundamentos históricos, filosóficos e metodológicos do campo. As produções foram analisadas por domínio, construindo-se assim uma cartografia preliminar do campo e apresentandose suas tendências e desafios.
\end{abstract}

PALAVRAS-CHAVE: Terapia ocupacional/tendências. Processo saúde-doença. Assistência hospitalar. Serviço hospitalar de terapia ocupacional hospitalar. Política de saúde.

* Artigo produto de projeto de pesquisa: Práticas hospitalares em terapia ocupacional: um estudo sobre a consolidação do campo no Estado de São Paulo, que a pesquisadora vem desenvolvendo com o apoio do CNPq.

1. Professora doutora, docente do Departamento de Fisioterapia, Fonoaudiologia e Terapia Ocupacional da Faculdade de Medicina da USP.

Endereço para correspondência: Centro de Docência e Pesquisa em terapia Ocuapcional da FMUSP, Rua Cipotânea, 51 - Cidade Universitária, 05508-900, São Paulo-SP. 


\section{INTRODUÇÃO}

A análise de produção científica é um dos recursos utilizados para se conhecer a constituição de um campo, suas tendências, bases conceituais e metodológicas. Artigos científicos e textos em geral refletem uma determinada realidade assim como a constroem. A compreensão da realidade, portanto, se dá por aquilo que a produção revela e pelo o que oculta ou não deixa transparecer. É possível também compreender como tal produção se dá frente à divisão social do saber e do trabalho, às concepções de saúde e às políticas do setor.

A importância da análise de produção científica sobre a intervenção do terapeuta ocupacional nos processos saúdedoença, principalmente aqueles ligados aos transtornos orgânicos, que requerem cuidados de clínica médica e cirúrgica é a de trazer subsídios para o entendimento da constituição do campo recentemente referido como campo das práticas hospitalares em terapia ocupacional (ou contextos hospitalares, como preferem alguns).

A intervenção do terapeuta ocupacional no hospital mudou significantemente de sua origem asilar para a atual configuração do hospital de agudos. Entretanto, a incorporação do profissional como essencial na equipe de saúde do hospital ainda não está consolidada. Questionamentos se colocam sobre o diálogo que a terapia ocupacional estabelece com a atenção hospitalar, sua interface com a atenção à pessoa doente realizada nos demais equipamentos de saúde e com a produção da saúde propriamente dita.

Afinal, que conhecimentos a terapia ocupacional incorpora neste diálogo? Como ela organiza seu discurso? Ela segue a racionalidade da especialidade médica ou a da saúde coletiva? Mistura ambas? Organiza sua intervenção pelo enfoque da reabilitação ou busca também incorporar a concepção da promoção da saúde? Como entende os processos saúde-doença: pela perspectiva naturalizante da doença ou pela perspectiva da doença enquanto produção social?

Compreender o uso de seus referenciais pode vir a ajudar a se estabelecer uma cartografia do campo das práticas hospitalares em terapia ocupacional e contribuir para a compreensão das contradições e dilemas do campo no que se refere a sua denominação, às concepções de saúde que adota, sua interlocução com os demais segmentos do setor saúde e, enfim ao papel da terapia ocupacional nas diferentes práticas. $\mathrm{O}$ estudo da produção científica do campo pode ser um passo inicial à discussão de sua cartografia. Assim, este artigo se propõe a apresentar uma perspectiva global da produção científica brasileira sobre as práticas hospitalares em terapia ocupacional, de 1990 a 2006, por domínio e temática.

\section{Sobre os procedimentos metodológicos}

Foi realizado um amplo levantamento bibliográfico da produção no país, de 1990 a 2006, encontrada na forma de artigos em periódicos indexados e não indexados ${ }^{(1)}$, capítulos de livro e resumos ampliados dos Anais do V Congresso Brasileiro e IV Simpósio Latino-Americano de Terapia Ocupacional (1997) e o IX Congresso Brasileiro de Terapia Ocupacional (2005).

Para o levantamento dos artigos indexados, foram consultadas as bases de dados LILACS, MEDLINE e SciELO, usando-se o descritor serviço hospitalar de terapia ocupacional e combinações dos descritores terapia ocupacional, hospitalização e assistência hospitalar. A seguir, outras combinações com o descritor terapia ocupacional foram exploradas, e na medida em que novos descritores eram identificados nos textos, eles passavam a incorporar o repertório de busca. Ao final foram utilizados em combinação com terapia ocupacional os seguintes descritores: criança hospitalizada, adolescente hospitalizado, neoplasias, doenças reumáticas, unidade de terapia intensiva neonatal, alojamento conjunto, ginecologia, neonatologia, pediatria, ortopedia, neurologia, oncologia, nefrologia, geriatria, clínica médica, clínica cirúrgica e unidade de queimados.

O corpus gerado pelo levantamento bibliográfico consistiu de 86 registros de produções, cujos dados foram organizados em um banco de dados, que vem sendo analisado sob diferentes recortes: por domínio e temática, tipos de veículos de divulgação e sua indexação, procedência dos autores, regionalização do trabalho, palavras-chave, descritores, referências bibliográficas e conteúdo. Este montante não perfaz o universo das produções do campo, mas representa uma cobertura bastante compreensiva.

Destes 86 registros, dois são livros que têm significante interface da terapia ocupacional com a atenção hospitalar, e, por este motivo, foram contados individualmente assim como seus capítulos específicos. Para esses livros, o critério da seleção dos capítulos foi a de que os

\footnotetext{
(1) No caso dos periódicos Cadernos de Terapia Ocupacional da UFSCar e Revista Prática Hospitalar, que não têm mecanismo de busca, foi feita varredura manual.
} 
textos fossem de terapia ocupacional e se referissem ao trabalho em algum programa hospitalar (atendimento em enfermaria, ambulatório, atendimento domiciliário). Para não haver duplicidade na contagem, a análise da produção bibliográfica por domínio e temática faz referência apenas aos capítulos selecionados, o que reduz o corpus de análise para 84 registros. Desses, 25 são de resumos ampliados em anais de congresso, que começam a figurar como produção referida e serão levados em conta nesta análise ${ }^{(2)}$.

\section{A produção do conhecimento no campo: uma in- trodução}

O primeiro aspecto de destaque da análise da produção científica no campo da terapia ocupacional nas práticas/contextos hospitalares é o não reconhecimento desta terminologia como descritora da produção do campo da intervenção da terapia ocupacional junto a pessoas sob cuidados clínicos e cirúrgicos. Do levantamento do descritor serviço hospitalar de terapia ocupacional, cinco artigos nacionais podem ser identificados, sendo três sobre hospitais psiquiátricos e dois sobre programas de saúde do trabalhador em hospitais. A associação dos termos terapia ocupacional e hospitalização, seja como descritores ou palavras, não gera mais do que duas produções. Já na associação com assistência hospitalar nenhum resultado é encontrado. Na consulta às bases de dados por meio das palavras terapia ocupacional e hospital, 25 produções nacionais aparecem, das quais a grande maioria é sobre o hospital psiquiátrico. Apenas 4 se referem à atuação do terapeuta ocupacional em hospital geral. Portanto, a localização da produção em periódicos foi a partir dos descritores mencionados na metodologia e pela busca em referências bibliográficas.

Os artigos são pouco referenciados tanto nos livros quanto nas produções em congressos, talvez por sua difícil localização. Há apenas dois livros específicos sobre a atuação da terapia ocupacional no hospital de autoria nacional: um sobre a atuação em pediatria (KUDO et al., 1994) e outro sobre as práticas hospitalares em geral (DE CARLO; LUZO, 2004), que concentram a maioria dos capítulos registrados por este estudo.

Estes livros e alguns resumos ampliados publicados em anais de congressos representam as principais referências específicas de terapia ocupacional em contextos hospitalares encontradas nos trabalhos publicados no campo, que em geral, apresentam-se como relato de experiência ou relato de estudo clínico. Há que se mencionar que esses trabalhos também referem outros campos da terapia ocupacional (Fundamentos, Saúde Mental) ou de outros campos de conhecimento (Clínicas Médicas, Psicologia, Fisiologia) para estabelecerem suas bases conceituais. Estes resultados, entretanto, são parciais e requerem estudos mais detalhados para a compreensão dos referenciais do campo.

\section{A produção do conhecimento no campo e seus domínios}

Como a proposta deste artigo é a busca da configuração do campo pelas temáticas, palavras-chave e descritores utilizados, a análise preliminar buscou agrupá-los por domínios, a partir do assunto e da freqüência de seu aparecimento. O resultado é o agrupamento da produção do campo em cinco domínios (Tabela 1), a saber: (a) atenção à gestante, puérpera e neonato; (b) atenção à criança e ao adolescente em enfermarias pediátricas; (c) a atenção ao adulto e ao idoso em hospital geral; (d) atenção às pessoas com câncer e/ou HIV/AIDs; (e) fundamentos históricos, filosóficos, metodológicos do campo.

\section{A produção referente às intervenções com gestantes, puérperas e neonatos}

A produção sobre as intervenções com gestantes, puérperas e neonatos no hospital retrata o movimento de cuidar da saúde da mulher, da gestante e do recém-nato precocemente, exercendo um fator adicional de proteção à saúde materno-infantil. A produção consiste de relatos de experiência, em sua maioria, distribuídos em seis artigos, dois capítulos de livro e oito resumos ampliados. A intervenção da terapia ocupacional em trabalho corporal com adolescentes, atendimentos à gestante ou em casos de gravidez de risco, apesar de serem intervenções em geral realizadas na rede básica de saúde, aparecem em produções sobre o atendimento em ambulatórios de hospitais (ANDRADE; SANTOS, 2004; DOMINGUES, 1992). Baseadas nos princípios da promoção à saúde e buscando a melhoria dos índices de morbi-mortalidade materno-infantil, as intervenções no âmbito hospitalar se referem, em geral, a mulheres em situação de vulnerabilidade ${ }^{(3)}$ por pobreza; mánutrição; baixo acesso a acompanhamento pré e perinatal; gravidez precoce ou tardia; violência doméstica ou sexual, e exposição a doenças sexualmente transmissíveis.

\footnotetext{
(2) Apenas alguns resumos serão referidos neste artigo, dado o dimensionamento do mesmo.

(3) Para melhor compreensão do conceito de vulnerabilidade ver AYRES et al., 2004.
} 
GAlHeIGO, S. M. Domínios e temáticas no campo das práticas. Rev. Ter. Ocup. Univ. São Paulo, v.18, n. 3, p. 113-121, set./dez. 2007.

Tabela 1 - Produção no campo das práticas hospitalares por temática e volume de produção de 1988 a 2006

\begin{tabular}{|c|c|c|c|}
\hline Domínios & Temáticas & $\begin{array}{l}\text { Registros por } \\
\text { temática }\end{array}$ & $\begin{array}{l}\text { Registros por } \\
\text { domínio }\end{array}$ \\
\hline \multirow{2}{*}{$\begin{array}{l}\text { Atenção à gestante, } \\
\text { puérpera e neonato }\end{array}$} & Gineco-obstetrícia & 3 & \multirow{2}{*}{$\begin{array}{c}16 \\
(6 \mathrm{~A} ; 2 \mathrm{C} ; 8 \mathrm{R})\end{array}$} \\
\hline & Neonatologia & 13 & \\
\hline \multirow{3}{*}{$\begin{array}{l}\text { Atenção à criança e ao } \\
\text { adolescente em enfer- } \\
\text { marias pediátricas }\end{array}$} & Pediatria & 21 & \multirow{3}{*}{$\begin{array}{l}24 \\
\text {;0C; 4R) }\end{array}$} \\
\hline & Humanização do hospital/enfermaria pediátricos & 2 & \\
\hline & Humanização/ambulatório/brinquedoteca & 1 & \\
\hline \multirow{6}{*}{$\begin{array}{l}\text { Atenção ao adulto e ao } \\
\text { idoso em hospital geral }\end{array}$} & Hospital geral & 10 & \multirow{6}{*}{$\begin{array}{l}25 \\
5 \mathrm{C} ; 11 \mathrm{R})\end{array}$} \\
\hline & $\begin{array}{l}\text { Abordagens especiais: pessoa ostomizada; pessoa } \\
\text { com obesidade mórbida; manejo da dor }\end{array}$ & 3 & \\
\hline & Interface com a reabilitação: neurologia & 3 & \\
\hline & Interface com a reabilitação: ortopedia & 3 & \\
\hline & Interface com a reabilitação: reumatologia & 3 & \\
\hline & Interface com a reabilitação: queimados & 3 & \\
\hline \multirow{5}{*}{$\begin{array}{l}\text { Atenção às pessoas com } \\
\text { câncer e/ou HIV/AIDs }\end{array}$} & Oncologia & 11 & \multirow{5}{*}{$\begin{array}{c}16 \\
\mathrm{~A} ; 2 \mathrm{C} ; 2 \mathrm{R})\end{array}$} \\
\hline & Ginecologia/câncer de mama & 1 & \\
\hline & HIV/AIDs & 2 & \\
\hline & HIV/AIDs/Pediatria & 1 & \\
\hline & HIV/AIDs/ Cuidados paliativos & 1 & \\
\hline \multirow{2}{*}{$\begin{array}{l}\text { Fundamentos históricos, } \\
\text { filosóficos, metodoló- } \\
\text { gicos }\end{array}$} & Fundamentos históricos e metodológicos & 1 & \multirow{2}{*}{$\begin{array}{c}2 \\
(2 \mathrm{C})\end{array}$} \\
\hline & Discussão filosófica sobre o corpo & 1 & \\
\hline Total & & & $\begin{array}{c}83 \\
(37 \mathrm{~A} ; 21 \mathrm{C} ; 25 \mathrm{R})\end{array}$ \\
\hline
\end{tabular}

A - Artigo em periódico; C - Capítulo de Livro; $\mathrm{R}$ - Resumo ampliado

Assim, as produções registram a participação do terapeuta ocupacional em iniciativas compartilhadas com a equipe multiprofissional de saúde, que começam ainda no hospital e continuam nos ambulatórios de seguimento. Dentre estas ações encontra-se o trabalho em alojamento conjunto, que também oferece atenção integral ao binômio mãe-filho, através de orientação materna sobre saúde, cuidados e aleitamento (PINTO et al., 1998). Mais recentemente, programas como o Hospital Amigo da Criança, idealizado pela OMS e pelo UNICEF em 1990, aparece nas referências sobre as atividades de promoção, proteção e apoio ao aleitamento materno desenvolvidas por terapeutas ocupacionais.

Em relação à neonatologia, o trabalho junto ao neonato pré-termo e/ou de risco se intensificou devido aos avanços tecnológicos que aumentaram seu índice de sobrevivência. A produção científica neste domínio tem registrado a riqueza das abordagens possíveis, envolvendo a intervenção direta com o neonato, o acompanhamento familiar e a orientação pós-alta em unidades de tratamento intensivo neonatal, nos berçários de cuidados especiais e intermediários e no acompanhamento ambulatorial (ARBAITMAN, 1994; MEYERHOF, 1994, 1997; OBANA; OSHIRO, 2002; DITZ et al., 2006;). A produção recente em congressos demonstra um novo tipo de intervenção do terapeuta ocupacional no hospital, a partir da implantação da Norma de Atenção Humanizada ao Recém-Nascimento de Baixo Peso (Método Mãe Canguru) pelo Ministério da Saúde desde 2002.

\section{A produção referente às intervenções com crianças e adolescentes hospitalizados}

Uma das áreas que vem tendo destaque em práticas consolidadas e produções acadêmicas é a atenção a crianças e adolescentes em enfermarias e unidades de terapia intensiva pediátricas (UTI). Há textos relevantes para a fundamentação da intervenção do terapeuta ocupacional neste domínio, que aparecem sob a forma de 10 artigos, 4 
resumos ampliados e 10 capítulos, sendo a maioria destes de um único livro (KUDO et al., 1994). O enfoque principal é no trabalho do terapeuta ocupacional na enfermaria e UTI, na preparação de crianças para cirurgias, e no acompanhamento familiar (KUDO et al, 1994; FERREIRA, 1997; ROSSIT; KOVACS, 1998; ALMEIDA et al., 2000; HIRSCHHEIMER et al., 2001; MITRE, 2002; MITRE; GOMES, 2004; TAKATORI et al., 2004). A maioria destes trabalhos e de resumos ampliados em anais de Congressos Brasileiros $(1997,2005)$ destaca a importância da atividade lúdica infantil no cotidiano hospitalar. Mais recentemente, a importância do brincar tem sido associada à discussão do papel da terapia ocupacional na brinquedoteca hospitalar, associada, em geral, às propostas de humanização do cuidado, em enfermarias pediátricas ou em salas de espera de ambulatórios de especialidade (MORENO et al, 2003; MITRE, 2006; MARIA; KUDO, 2006; SANTOS et al., 2006; THINEN et al., 2006).

\section{A produção referente às intervenções em hospital geral junto ao adulto e ao idoso}

A produção sobre a terapia ocupacional no hospital geral consiste de nove artigos, cinco capítulos de livro e 11 resumos ampliados. Tratam-se, em geral, de relatos de experiências de terapia ocupacional em hospitais gerais, em sua maioria, universitários. Os trabalhos apresentam as bases conceituais e as práticas desenvolvidas em diferentes enfermarias e programas e, ressaltam a importância do envolvimento do ensino e da formação continuada para o desenvolvimento dos serviços (PALM, 1997; MORAIS 2001; MOTTA; FERRARI, 2004; BIGATÃO et al., 2005; DE CARLO et al., 2006).

Pela produção levantada, a atuação do terapeuta ocupacional junto a adultos e idosos hospitalizados aparece nas enfermarias de clínica médica (cardiologia, endocrinologia, gastro-enterologia, geriatria, neurologia, nefrologia, oncologia, pneumologia e reumatologia), de clínica cirúrgica e de doenças infecciosas. Entretanto, não há produções específicas para as diferentes clínicas seguindo o padrão de especialidade médica. Há, ao contrário, uma tendência de se dar ao trabalho um caráter geral, para o hospital como um todo ao invés de trabalhos para enfermarias específicas, ocorrendo uma prática usual de interconsultas ou desenvolvimento de programas genéricos. Os programas de atendimento domiciliário (PAD) e de atenção ao cuidador figuram entre estes últimos e aparecem na produção da área dentro do contexto do trabalho no hospital.

Começa a surgir produção científica relativa aos impactos dos processos saúde-doença específicos, tais como a qualidade de vida da pessoa ostomizada (NOSELLA et al., 2006) ou com obesidade mórbida (MORAIS et al., 2002) e sobre abordagens específicas no manejo da dor (OLINTO et al., 2005). Entretanto, sua pulverização nos periódicos especializados dificulta um levantamento bibliográfico mais fidedigno, principalmente quando se tratam de ações compartilhadas com outros profissionais e o termo terapia ocupacional não consta no título ou descritores.

Há uma produção de difícil classificação e levantamento que é a referente à interface da reabilitação com os programas hospitalares. $\mathrm{O}$ atendimento domiciliário, ambulatorial e/ou comunitário que tem caracterizado a atenção à pessoa com deficiência é resultante do processo de desospitalização que fez o hospital de crônicos dar lugar ao hospital de agudos. Assim, a produção referente à terapia ocupacional com pessoas com seqüelas de queimaduras ou pessoas com transtornos neurológicos, reumatológicos e ortopédicos nem sempre discrimina a intervenção hospitalar dos demais tipos de intervenção. Para caracterizar então essa diferença, a pesquisa em questão classificou como hospitalar, quando havia no texto referência à atenção na fase aguda ou de exacerbação da doença tratado em ambiente hospitalar ou quando havia referência a atendimento ambulatorial ou domiciliário oferecido em dependências hospitalares. O livro Terapia ocupacional: reabilitação fisica e contextos hospitalares provê importante produção desta interface, totalizando 4 capítulos que contemplam as especialidades de neurologia, reumatologia, traumato-ortopedia e queimados (DE CARLO et al., 2004). Além da produção do livro, há mais dois resumos ampliados em neurologia; 1 resumo ampliado e 1 artigo em traumato-ortopedia; dois artigos em reumatologia e um artigo e um resumo ampliado em queimados (REIS et al., 1990; SIQUEIRA; JULIBONI, 2000; NOORDHOEK; LOSCHIAVO, 2004, 2005; CONGRESSO BRASILEIRO.., 2005). Estes resultados não condizem com o esperado em volume de produção e credita-se esta situação a uma dispersão da produção em periódicos e livros de reabilitação, que não foram o foco desta pesquisa.

A humanização hospitalar começa a ser referida em alguns dos textos acima citados. Entretanto, dada que a Política Nacional de Humanização do Ministério da Saúde, já vem sendo implementada no país desde 2004, há ainda baixa produção no assunto. $\mathrm{O}$ mesmo acontece em relação à explicitação da correlação das práticas hospitalares em terapia ocupacional com sua inserção no SUS, comum nas produções sobre a ação da terapia ocupacional nos programas desenvolvidos na rede básica de atenção à saúde, na reabilitação de pessoas com deficiência e na saúde mental. Entretanto, ela é ainda incipiente na produção de práticas hospitalares em terapia ocupacional, chegando a parecer 
que estas acontecem foram de um contexto mais amplo das políticas de saúde do país.

\section{A produção referente à atenção às pessoas com câncer e/ou HIV/AIDs}

Merece destaque a produção referente à atenção às pessoas com câncer e/ou HIV/AIDs. Apesar de algumas destas intervenções estarem incluídas nas produções sobre a terapia ocupacional no hospital geral de adultos e em pediatria, elas começam a se mostrar de forma consistente como um domínio à parte, o que norteia a proposta de separá-las da produção do hospital geral. As produções sobre a atuação da terapia ocupacional em oncologia somam 8 artigos, 2 capítulos de livro e 2 resumos ampliados. Destes 12 registros, 7 são sobre a intervenção da terapia ocupacional com pessoas que sofreram transplante de medula óssea. (MASTROPIETRO, 2001; MASTROPIETRO et al., 2003; CONGRESSO BRASILEIRO.., 2005; OLIVEIRA et al., 2003, 2005; MASTROPIETRO et al., 2006). Um trabalho é sobre a intervenção da terapia ocupacional em oncologia (OLIVEIRA et al. 2003) e outro, com mulheres com câncer tratadas em enfermaria de ginecologia (OBANA et al., 2006) Três trabalhos são sobre o trabalho da terapia ocupacional em oncologia com crianças (MORAIS; VALLE, 2001; PENGO; SANTOS 2004; VASCONCELOS et al., 2006),

As produções sobre a atenção da terapia ocupacional às pessoas com HIV/AIDS, embora ainda em menor número já mostra um potencial de crescimento, somando 4 produções. Há estudos sobre o perfil dos atendidos pela terapia ocupacional (JENTOF; QUEIROZ, 2002), sobre cuidados paliativos (AIRES et al., 2005) e dois sobre a atenção a crianças portadores de HIV/AIDS (ARTIGIANI, 1996; NEGRINI et al., 2005).

\section{A produção referente aos fundamentos, históricos, filosóficos e metodológicos do campo}

Há uma vasta produção bibliográfica na terapia ocupacional que, ao resgatar sua história e seus fundamentos, históricos, filosóficos e metodológicos retoma os processos de adoecimento, de hospitalização/ desospitalização/ desinstitucionalização, das intervenções sobre o corpo e da sujeição da pessoa aos cuidados médicos. Fazer um levantamento amplo do assunto retiraria o foco deste trabalho, parecendo suficiente mencionar apenas alguns títulos que concentram os esforços de reflexão e retrospectiva histórica no campo das práticas hospitalares (DE CARLO et al., 2005; ROCHA; MELLO, 2005).

\section{CONSIDERAÇÕES FINAIS}

Se a revisão da literatura aponta para um importante potencial de trabalho no campo das práticas hospitalares, ela demonstra uma fragilidade na sua constituição dado ao ainda pequeno número de artigos e livros. Poucas das produções analisadas eram relativas a resultados de pesquisa, sendo a maioria composta de relatos de experiência e alguns textos de caráter didático. Este recorte de análise mostra também que a produção se concentra em alguns domínios e sub-domínios que acabam por utilizar arcabouços teóricos distintos e dialogam com diferentes atores do setor saúde organizados por suas especialidades.

A produção em neonatologia e pediatria mostra um perfil mais constante, anteriormente fundado na promoção do desenvolvimento infantil através do brincar, mas, que recentemente tem se revigorado com as perspectivas trazidas pelo aporte da promoção de saúde, da integralidade das ações de saúde e dos recentes investimentos na humanização do cuidado trazido pela Política Nacional de Humanização. A produção sobre o hospital geral de adultos, seja em relação ao trabalho em enfermarias, ambulatórios ou programas de atendimento domiciliário, ainda apresenta uma dispersão por especialidades e uma produção insuficiente em artigos, embora tenha havido um aumento de produção em resumos ampliados em congressos. Há pouco diálogo da produção deste domínio com a política de saúde e as propostas de humanização do cuidado, sendo ainda o relato de experiências pontuais o que caracteriza a produção do domínio.

A produção sobre o trabalho em oncologia é o que vem mais crescendo, com larga produção sobre a intervenção da terapia ocupacional com pessoas que sofreram transplante de medula óssea, a maior parte dela de um mesmo grupo de pesquisa. A produção em relação à oncologia em geral e ao atendimento de crianças com câncer começa também a ganhar forma, embora seja ainda pulverizado. O mesmo pode-se dizer em relação à produção referente à HIV/AIDS que, embora crescente, é limitada se considerarmos a experiência acumulada na área. O domínio da discussão históricoepistemológica um dos que mais carecem de investimento, apesar do esforço dos dois capítulos publicados. A produção sobre a desinstitucionalização e a desospitalização, que não foi coberta por este artigo, ainda continua sendo a principal referência deste domínio.

Esta revisão é fruto de um estudo exploratório sobre o tema sobre o qual versa este projeto de pesquisa. Entretanto, resultados interessantes podem ser conseguidos através de uma análise da produção científica específica dos diferentes domínios. Através de análise de conteúdo, o percurso da terapia ocupacional nas práticas hospitalares poderá ser 
melhor delineado e seus dilemas melhor entendidos. Esforços que para a consolidação do campo são necessários e bem-vindos se quisermos contribuir para o cuidado das pessoas com transtornos em sua saúde.

GALHEIGO, S. M.Domains and themes in the field of occupational therapy in hospital practice: a literature review of Brazilian production from 1990 to 2006. Rev. Ter. Ocup. Univ. São Paulo, v.18, n. 3, p. 113-121, set./dez. 2007.

\begin{abstract}
This paper intends to present the literature review on the field of occupational therapy in hospital practice by domains and themes through a study of scientific production from 1990 to 2006. Papers, chapters of books, and extended abstracts of two Brazilian Occupational Therapy Congresses were review, adding up to 83 items. Data were analyzed according to four assistance domains: pregnancy; delivery and newborn; child and adolescent in pediatric wards; adults and elders in hospital care; people with cancer and HIV/AIDs and; historical, philosophical and methodological foundations. Literature review was undertaken by domain in order to build a preliminary map of the field and present its tendencies and challenges.
\end{abstract}

KEY WORDS: Occupational therapy/tendencies. Health-disease process. Hospitalar assistance. Hospitalar occupational therapy service. Health policy.

\title{
REFERÊNCIAS
}

AIRES, E. M., et al. Cuidados paliativos em pacientes com HIV/ Aids. Rev. Prática Hosp., São Paulo, n. 37, 2005. Disponível em: http://www.praticahospitalar.com.br/pratica\%2037/paginas/ materia\%2007-37.html. Acesso: 13 dez. 2006.

ALMEIDA, A., et al. Projeto brincar: a importância do brincar na intervenção hospitalar. Arq. Neuropsiquiatr., v. 58, p. 11, 2000.

ANDRADE, F. L.; SANTOS, S. C. Gravidez de alto risco - algumas possibilidades de atuação do terapeuta ocupacional. Rev. Ter. Ocup. Bahiana, v.1, n. 1, p. 20-24, 2004.

ARBAITMAN, E. Terapia ocupacional como agente facilitador na interação criança-família. In: KUDO et al. (orgs.). Fisioterapia, fonoaudiologia e terapia ocupacional em pediatria. 2a. ed. São Paulo: Sarvier, 1994. p. 223-231.

ARTIGIANI, G. Terapia ocupacional em AIDS pediátrica: trabalho desenvolvido na Unidade de Apoio Infantil, vinculado ao Centro Corsini - Campinas, SP. Cad. Ter. Ocup.. UFSCar, v. 5, n. 2, p. 121-126, 1996.

AYRES et al. O conceito de vulnerabilidade e as práticas de saúde: novas perspectivas e desafios. In: CZERESNIA, D.; FREITAS, C. M. (org.). Promoção da saúde: conceitos, reflexões, tendências. Rio de Janeiro: FIOCRUZ, 2003. p. 117-139.

BARICHELO et al. O uso de atividades lúdicas no processo de revelação do diagnóstico a crianças que vivem com HIV/AIDS. Cad. Ter. Ocup. UFSCar, v.14, n. 2, p. 111-119, 2006.

BIGATÃO, M.; PRIETO, G.; PALM, R. Relato de experiência do Programa de Aprimoramento Profissional em Terapia Ocupacional. In: CONGRESSO BRASILEIRO DE TERAPIA OCUPACIO-
NAL, 9., 2005, Recife. Anais.. Recife: ATOPE/ABRATO, 2005. CD-Rom.CD-Rom.

CONGRESSO BRASILEIRO, 5., SIMPÓSIO LATINO-AMERICANO DE TERAPIA OCUPACIONAL, 4., Belo Horizonte, 1997. Anais.. Belo Horizonte: ATOPE/ABRATO, 1997.

CONGRESSO BRASILEIRO DE TERAPIA OCUPACIONAL, 9., 2005, Recife. Anais.. Recife: ATOPE/ABRATO, 2005. CD-Rom.

DE CARLO, M., et al. Terapia ocupacional em contextos hospitalares. Rev. Prática Hosp. São Paulo, n. 43, p.158-164, 2006.

DE CARLO, M.; BARTALOTTI, C.; PALM, R. A terapia ocupacional em reabilitação física e contextos hospitalares: fundamentos para a prática. In: DE CARLO, M.; LUZO, M. C. (orgs.). Terapia ocupacional: reabilitação física e contextos hospitalares. São Paulo: Rocca, 2004. p. 3-28.

DE CARLO, M.; LUZO, M. C. (orgs.). Terapia ocupacional: reabilitação física e contextos hospitalares. São Paulo: Rocca, 2004.

DITTZ, E.; MELO, D.; PINHEIRO, Z. A terapia ocupacional no contexto da assistência à mãe e à família de recém-nascidos internados em unidade de terapia intensiva. Rev. Ter. Ocup. Univ. São Paulo, v. 17, n. 1, p. 42-47, 2006.

DOMINGUES, J. Aspectos da Intervenção de Terapia Ocupacional no Ambulatório de Ginecologia de Adolescentes. Rev. Ter. Ocup. Univ. São Paulo, v. 3, n. 1/2, p. 65-71, 1992.

FERREIRA, L. Terapia ocupacional: uma experiência em UTI pediátrica. Rev. Ter. Ocup. PUCCAMP. Campinas, n. ed. esp., p. 32-34. 1997. 
GAlHEIGO, S. M. Domínios e temáticas no campo das práticas. Rev. Ter. Ocup. Univ. São Paulo, v.18, n. 3, p. 113-121, set./dez. 2007.

HIRSCHHEIMER et al. O trabalho da terapia ocupacional na pediatria. Rev. Paul. Pediatr., v. 19, n. 4, p. 187-194, 2001.

JENTOF, I.; QUEIROZ, M. A Terapia ocupacional e AIDS: atuação no Instituto de Infectologia Emílio Ribas, São Paulo, Brasil. Cad. Centro Univers. São Camilo, v. 8, n. 3, p. 44-47, 2002.

KUDO, A., et al. (orgs.). Fisioterapia, fonoaudiologia e terapia ocupacional em pediatria. 2a. ed. São Paulo: Sarvier, 1994.

MARIA, P.; KUDO, A. Intervenção da terapia ocupacional em pediatria. In: SILVA, A.P., et al. O Instituto da Criança - 30 Anos - ações atuais na atenção interdisciplinar em pediatria. São Paulo: Editora Yendis, 2006. p. 211-235.

MASTROPIETRO, A. P. Implantação de um serviço de terapia ocupacional em uma unidade de transplante de medula óssea. Centro de Estudos de Terapia Ocupacional, São Paulo, 2001. v. 6.

MASTROPIETRO, A. P., et al. Reestruturando a vida após o transplante de medula óssea: reinserção ocupacional de pacientes transplantados. In: MELO-SILVA, L. L., et al. (org.). Arquitetura de uma ocupação: orientação profissional: teoria e prática. São Paulo: Vetor Psicopedagógica Ltda, 2003. p. 227-236.

MASTROPIETRO, A. P.; SANTOS, M.; OLIVEIRA, E. Sobreviventes do transplante de medula óssea: construção do cotidiano. Rev. Ter. Ocup. Univ. São Paulo, v. 17, p. 64-17, 2006.

MEYERHOF, P. O neonato de risco: proposta de intervenção no ambiente e no desenvolvimento. In: KUDO et al. (orgs.), Fisioterapia, fonoaudiologia e terapia ocupacional em pediatria. 2a.ed. São Paulo: Sarvier, 1994. p. 204-222.

MEYERHOF, P. O neonato pré-termo no berçário de cuidados especiais. Temas Sobre Desenvolvimento, v. 5, n. 30, p. 15-19, 1997.

MITRE. R. O papel do brincar na hospitalização de crianças: uma reflexão. Pediatr. Mod., v. 7, n. 38, p. 339-342, 2002.

MITRE, R. O brincar no processo de humanização da produção de cuidados pediátricos. In: DESLANDES, S. (org.). Humanização dos cuidados e saúde: conceitos, dilemas e práticas. Rio de Janeiro: FIOCRUZ, 2006. p. 283-300.

MITRE, R.; GOMES, R. A promoção do brincar no contexto da hospitalização infantil como ação de saúde. Ciênc. Saúde Coletiva, v. 9, n. 1, p.147-154, 2004.

MORAIS, L. A interconsulta de terapia ocupacional no hospital geral: um espaço para a saúde. Rev. Centro Estudos Ter. Ocup., n. 6 , p. 9-13, 2001.

MORAIS, L., et al. O cotidiano na obesidade mórbida : um espaço para a terapia ocupacional. Rev. Diagn. Tratamento, v. 7, n. 4, p. 18-21, 2002.

MORAIS, L.; VALLE, M. R. A terapia ocupacional e a criança hospitalizada para tratamento de câncer : relato de um atendimento. Rev. Pediatr. Mod., v. 37, p. 177-183, 2001.
MORENO, R. L., et al. Contar histórias para crianças hospitalizadas: relato de uma estratégia de humanização. Pediatria (São Paulo), v. 25, n. 4, p. 164-169, 2003.

MOTTA, M.; FERRARI, M. A. Intervenção terapêutico-ocupacional junto a indivíduos com comprometimento no processo de envelhecimento. In: DE CARLO, M.; LUZO, M.C. (orgs.). Terapia ocupacional: reabilitação física e contextos hospitalares. São Paulo: Rocca, 2004. p. 233-255.

NOORDHOEK, J.; LOSCHIAVO, F. Adaptação visando melhora do desempenho funcional em atividade da vida diária para adolescentes com artrite reumatóide juvenil. Rev. Bras. Reumatol., v. 44, n. 5, p. 362-3, 2004.

NOORDHOEK, J.; LOSCHIAVO, F.Q. Intervenção da terapia ocupacional no tratamento de indivíduos com doenças reumáticas utilizando a abordagem da proteção articular. Rev. Bras. Reumatol, v. 45 , n. 4 , p. 242-244, 2005.

NOSELLA, V.; MARTINS, M.; GOMES NETINHO, J. Qualidade de vida e atividades cotidianas dos pacientes ostomizados definitivos. Rev. Prática Hosp., São Paulo, n. 44, 2006. Disponível em: http://www.praticahospitalar.com.br/pratica\%2044/pgs/materia\%2007-44.html. Acesso: 13 dez. 2006.

OBANA, A et al. Terapia ocupacional na enfermaria de ginecologia: um enfoque na qualidade de vida durante a hospitalização. Mundo Saúde, v. 30, n. 1, p. 185-188, 2006.

OBANA, A.; OSHIRO, M. A Terapia Ocupacional com bebês de risco: reflexões sobre a clínica. Cad. Centro Univers. São Camilo, v.8, n. 3, p.58-61, 2002.

OLINTO, C., et al. Grupos educativos no manejo de pacientes com dor crônica. Rev. Prática Hosp., São Paulo, n. 41, 2005. Disponível em: http://www.praticahospitalar.com.br/ pratica\%20 41/pgs/materia\%2016-41.html. Acesso: 13 dez. 2006.

OLIVEIRA, A., et al. Reflexões sobre a prática de terapia ocupacional em oncologia na cidade de São Carlos. Cad. Ter. Ocup. UFSCAR, v. 11, n. 2, p.118-123, 2003.

OLIVEIRA, E., et al. Grupo de apoio ao familiar do transplantado de medula óssea. Rev. SPAGESP, v. 4, n. 4, p. 52-56, 2003.

OLIVEIRA, E., et al. Intervenção junto à família do paciente com alto risco de morte. Medicina (Ribeirão Preto), v. 38, n. 1, p. 63-68, 2005.

PALM, R. C. Construindo ações interdisciplinares em um hospital geral. In: CONGRESSO BRASILEIRO, 5., SIMPÓSIO LATINO-AMERICANO DE TERAPIA OCUPACIONAL, 4., Belo Horizonte, 1997. Anais. Belo Horizonte: ATOPE/ABRATO, 1997. p. 52-63.

PENGO, M.; SANTOS, W. O papel do terapeuta ocupacional em oncologia. In: DE CARLO, M.; LUZO, M. C. (orgs.). Terapia ocupacional: reabilitação física e contextos hospitalares. São Paulo: Rocca, 2004. p. 233-255. 
GALHEIGO, S. M. Domínios e temáticas no campo das práticas. Rev. Ter. Ocup. Univ. São Paulo, v.18, n. 3, p. 113-121, set./dez. 2007.

PINTO, L., et al. Caracterização e avaliação qualitativa do sistema de alojamento conjunto do Hospital e Maternidade Celso Pierro. Pediatria (São Paulo), v. 20, n. 4., p. 416-422, 1998.

REIS, et al. Terapia ocupacional no tratamento das fraturas do terço distal do rádio. Folha Med., 100, n. 1/2, p. 15-8, 1990.

ROCHA, E.; MELLO, M. A. Os sentidos do corpo e da intervenção hospitalar. DE CARLO, M.; LUZO, M.C. (orgs.). Terapia ocupacional: reabilitação física e contextos hospitalares. São Paulo: Rocca, 2004. p. 29-46.

ROSSIT, R.; KOVACS, A. Intervenção essencial em terapia ocupacional em enfermaria pediátrica. Cad. Ter. Ocup. UFSCar, v. 7, n. 2, p. 58-67, 1998.

SANTOS, C.; MARQUES, E.; PFEIFER, L.I. A brinquedoteca sob a visão da terapia ocupacional: diferentes contextos. Cad. Ter. Ocup. UFSCar, v.14, n. 2, p. 91-102, 2006.
SIQUEIRA, F.; JULIBONI, E. O papel da atividade terapêutica na reabilitação do indivíduo queimado em fase aguda. Cad. Ter. Ocup. UFSCar, v. 8, n. 2, p. 79-91, 2000.

TAKATORI, M.; OSHIRO, M.; OTASHIMA, C. O Hospital e a assistência em terapia ocupacional infantil. In: DE CARLO, M.; LUZO, M. C. (orgs.). Terapia ocupacional: reabilitação física e contextos hospitalares. São Paulo: Rocca, 2004. p. 257-275.

THINEN, N.; MORAES, A.C.; BARBOSA, M. Humanização do ambulatório de especialidades Governador Mario Covas: criação de uma brinquedoteca. Cad. Ter. Ocup. UFSCar, v. 13, .n. 2, p. 99-107, 2006.

VASCONCELOS, R.; ALBUQUERQUE, V.; COSTA, M. L. Reflexões da clínica terapêutica ocupacional junto à criança com câncer na vigência da quimioterapia. Rev. Bras. Cancerol., v. 52, n. 2, p. 129-137, 2006. 